\title{
À la gauche de Dieu. Une géographie de la contestation catholique en Italie dans les années 1960-1970
}

\author{
Marta Margotti
}

Il n'est pas facile de reconstruire la cartographie de la contestation catholique en Italie dans les années 1960 et 1970. La fragmentation des expériences, les différentes sources d'inspiration, les finalités et les réalisations concrètes, rendent le domaine à explorer plus semblable à un archipel méditerranéen qu'à un territoire continental homogène ${ }^{1}$. Comme pour d'autres réalités nationales, la définition de l'objet de la recherche s'avère particulièrement complexe, à commencer par les termes utilisés pour le décrire. Citons les principaux: contestation, dissidence, progressisme catholique, communautés de base, groupes spontanés, catholiques désobéissants, catholiques rebelles, chrétiens pour le socialisme ou, encore, catholiques communistes. À cette difficulté commune, s'ajoute la particularité de la situation religieuse et politique du pays : en effet, au-delà d'être le siège de la papauté, l'Italie $\mathrm{a}$, sans interruption, été dirigée, de 1945 à 1993, par des gouvernements qui intégraient des représentants de la Démocratie chrétienne, le parti modéré de majorité relative, auquel les institutions catholiques ont longuement apporté leur soutien, mais le pays a également été caractérisé par la présence d'un très fort Parti communiste, le plus puissant d'Europe occidentale.

Cette imbrication d'éléments politiques et religieux a profondément marqué le développement de la contestation catholique dans la péninsule italienne. En premier lieu, le pays a assisté à l'émergence d'une forte radicalisation des positions, principalement au sein des groupes dans lesquels s'enchevêtraient des revendications de réforme de l'Église et une volonté de renouvellement de la société. En second lieu, un degré élevé de conflictualité dans l'action sociale, également marqué par une aspiration à l'innovation théorique, a pu s'observer au sein des groupes nés à l'extérieur de la sphère d'influence des paroisses et des associations catholiques alors présentes sur le territoire. En tout cas, les idées que ces groupes défendaient ont connu une certaine diffusion et un écho considérable, précisément à l’intérieur des organisations de masse de l'Église italienne. En troisième lieu, au-delà de la surveillance constante des forces de police, il ne faut pas manquer de relever

\footnotetext{
${ }^{1}$ Cf. Attilio Agnoletto, "Gli anni del Concilio e del post-Concilio e il "dissenso cattolico" », dans Francesco Traniello et Giorgio Campanini (dir.), Dizionario storico del movimento cattolico in Italia. 186o-1980, tome I/1, Turin, Marietti, 1981, p. 112-121; Maurilio Guasco, Storia del clero in Italia dall'Ottocento a oggi, Rome-Bari, Laterza, 1997, p. 253-296 ; Guido Verucci, « Il dissenso cattolico in Italia ", Studi storici, tome 43, $\mathrm{n}^{\circ}$ 1, janvier-mars 2002, p. 215-233; Marco Impagliazzo, Il dissenso cattolico e le minoranze religiose, dans Fiamma Lussana et Giacomo Marramao (dir.), L'Italia repubblicana nella crisi degli anni Settanta, tome 2: Culture, nuovi soggetti, identità, Soveria Mannelli, Rubbettino, 2003, p. 231-251; Daniela Saresella, Cattolici a sinistra. Dal modernismo ai giorni nostri, Rome-Bari, Laterza, 2011, p. 98-165; Alessandro Santagata, La contestazione cattolica. Movimenti, cultura e politica dal Vaticano II al '68, Rome, Viella, 2016.
} 
Marta Margotti, «À la gauche de Dieu. Une géographie de la contestation catholique en Italie dans les années 19601970 », Histoire@Politique, [en ligne], n 30, septembre-décembre 2016, www.histoire-politique.fr

l'attention toute particulière, et non exclusivement religieuse, dont les différentes expressions de la contestation catholique furent l'objet de la part des formations politiques et des médias d'information dès lors qu'elles pouvaient entraîner des répercussions directes sur les équilibres politiques du pays.

Afin de s'orienter plus aisément dans cet archipel composé de plusieurs centaines de groupes et mouvements, nous proposons un certain nombre d'axes d'interprétation formant un cadre provisoire pour un sujet d'étude dont la complexité doit encore être inventoriée, à commencer par les sources archivistiques qui sont souvent dispersées ou partiellement accessibles aux chercheurs (notamment les archives ecclésiastiques et les sources policières) ${ }^{2}$. Il s'agit de plusieurs hypothèses de recherche qui, à l'image d'une boussole, permettent de suivre les transformations de la contestation catholique dans une Italie éprouvée par les tensions sociales, les crises économiques, les conflits politiques et la violence terroriste qui modifièrent en profondeur la physionomie du pays et, en certaines occasions, semblèrent même pouvoir ébranler son assise démocratique.

Il est possible de formuler l'hypothèse selon laquelle le dissenso cattolico (selon l'expression par laquelle on désigne habituellement la gauche catholique italienne) a pris des formes résolument radicales en raison de l'endiguement prolongé des aspirations favorables au renouveau religieux durant les années antérieures et, également, de l'enchevêtrement de phénomènes de protestation politique et sociale d'une grande virulence ayant essentiellement débuté au cours de la seconde moitié des années 1960. La montée de la contestation politique (du capitalisme et du monopole démocrate-chrétien du vote catholique) - à laquelle s'ajoutèrent la critique de l'autoritarisme ecclésiastique et l'espoir d'une réforme de l'Église - fit croître les tensions à l'intérieur de la communauté chrétienne. Celles-ci allèrent jusqu'à provoquer, dans certains cas, des mesures particulièrement sévères de la part de la hiérarchie catholique, qui favorisa pourtant indirectement le renouvellement des structures ecclésiales et du sentiment religieux des catholiques italiens.

Cette étude nous donnera l'occasion de présenter une proposition de périodisation de la dissidence catholique, un panorama à la fois topographique et culturel des chrétiens de gauche et un aperçu des rapports internationaux tissés par les différents groupes en présence. Certaines considérations mentionnées en conclusion nous permettront d'effectuer une comparaison entre la contestation catholique propre à l'Italie et les expériences développées dans d'autres pays, de manière à esquisser, in fine, une contribution à l'élaboration d'une histoire globale de la gauche catholique.

\section{Dynamiques et périodisation}

Pour appréhender les particularités et les dynamiques internes à la contestation italienne, il nous semble utile de proposer une périodisation ample, en mesure d'embrasser quelque vingt-cinq années d'histoire, depuis le milieu des années 1950 jusqu'au début des années 1980. Durant cette époque, le développement, la disparition ou la transformation d'une multitude d'expériences, pourtant minoritaires et souvent conduites en marge des institutions catholiques, laissèrent une empreinte durable sur l'ensemble de l'Église de la péninsule.

${ }^{2}$ Cf. Alessandro Santagata, « Una rassegna storiografica sul dissenso cattolico in Italia », Cristianesimo nella storia, tome $31, \mathrm{n}^{\circ} 1$, janvier-avril 2010, p. 207-241. 
Marta Margotti, « À la gauche de Dieu. Une géographie de la contestation catholique en Italie dans les années 19601970 », Histoire@Politique, [en ligne], n 30, septembre-décembre 2016, www.histoire-politique.fr

$\mathrm{Au}$ cours des dernières années du pontificat de Pie XII et durant la première partie de celui de Jean XXIII, des ferments de renouveau (déjà présents antérieurement, mais condamnés par le Saint-Siège), ainsi que des réflexions critiques portant principalement sur les formes de la présence de l'Église en Italie, se diffusent dans le clergé et dans le laïcat 3 . Certaines revues qui réfléchissaient sur le renouveau de l'Église et la nécessité d'un dialogue avec les non-croyants avaient suscité de fortes appréhensions au sein de la hiérarchie catholique. Il s'agissait, notamment, du mensuel génois Il Gallo, dirigé depuis 1946 par l'écrivain Nando Fabro, de la revue Adesso, fondée en 1949 par l'abbé Don Primo Mazzolari, curé d'une paroisse lombarde, ainsi que de la publication florentine Testimonianze dont le père Ernesto Balducci devint le directeur à partir de 19584. Des signes de mécontentement s'étaient également manifestés dans les secteurs de la jeunesse de l'ACI (Azione Cattolica Italiana/Action catholique italienne) et des ACLI (Associazioni Cristiane dei Lavoratori Italiani / Associations chrétiennes des travailleurs italiens) où se faisait jour une certaine impatience vis-à-vis d'une Église imperméable aux problématiques de la société moderne, mais aussi un rejet du "collatéralisme» entre les organisations catholiques et la Démocratie chrétienne.

Cette longue phase d'endiguement des aspirations favorables au renouveau catholique, exercé par une grande partie de l'épiscopat italien et par la curie vaticane, s'assouplit durant le pontificat de Jean XXIII et le Concile, qui permettent la fermentation au sein du catholicisme italien de suggestions spirituelles et d'initiatives originales, ainsi qu'une large circulation de nouvelles réflexions théologiques provenant souvent de l'étranger. La crise des organisations catholiques de masse est à la fois la cause et la conséquence de la multiplication de " groupes spontanés », qui se développent vers le milieu des années 1960 sur la base d'idéaux de pauvreté et d'engagement citoyen. Ces groupes remettent en cause le conformisme ecclésiastique, mais également les lenteurs de la réception du Concile, l'aridité spirituelle et les contradictions des structures catholiques. À ces critiques s'ajoutent bientôt des attaques contre l'unité politique des catholiques et contre la Démocratie chrétienne, accusée de se présenter comme le «parti catholique » par excellence et, surtout, de soutenir activement le capitalisme au détriment des classes populaires.

$\mathrm{Au}$ cours des années qui suivent immédiatement la fin du Concile, ces « catholiques de la diaspora » deviennent les animateurs de multiples débats et rassemblements publics qui, dans un certain nombre de cas, prennent directement appui sur des

3 Les choix de la petite paroisse de l'abbé Milani, située à Barbiana, sur les hauteurs des alentours de Florence, sont représentatifs de cette mouvance; cf. Giovanni Miccoli, "Don Lorenzo Milani nella Chiesa del suo tempo ", dans Fra mito della cristianità e secolarizzazione. Studi sul rapporto chiesasocietà nell'età contemporanea, Casale Monferrato, Marietti, 1985, p. 428-454. Sur le dialogue entre catholiques et communistes, cf. Mario Gozzini (dir.), Il dialogo alla prova. Cattolici e comunisti italiani, Florence, Vallecchi, 1964 ; Lorenzo Bedeschi, La sinistra cristiana e il dialogo con i comunisti, Parme, Guanda, 1966. Cf. Alberto Melloni, Da Giovanni XXIII alle chiese italiane del Vaticano II, dans Tullio Gregory, André Vauchez et Gabriele De Rosa (dir.), Storia dell'Italia religiosa, tome 3 : L'età contemporanea, Rome-Bari, Laterza, 1995, p. 361-403.

4 Cf. Giorgio Campanini et Matteo Truffelli (dir.), Mazzolari e Adesso cinquant'anni dopo, Brescia, Morcelliana, 2000 ; Bruna Bocchini Camaiani, Ernesto Balducci. La Chiesa e la modernità, Rome-Bari, Laterza, 2002 ; Daniela Saresella, Dal concilio alla contestazione. Riviste cattoliche negli anni del cambiamento (1958-1968), Brescia, Morcelliana, 2005; Paolo Zanini, La rivista "Il gallo". Dalla tradizione al dialogo (1946-1965), Milan, Biblioteca francescana, 2012 ; Daniela Saresella et Giorgio Vecchio (dir.), Mazzolari e il cattolicesimo prima del Concilio vaticano II, Brescia, Morcelliana, 2012 ; Mariangela Maraviglia, David Maria Turoldo, La vita, la testimonianza (1916-1992), Brescia, Morcelliana, 2016. 
Marta Margotti, « À la gauche de Dieu. Une géographie de la contestation catholique en Italie dans les années 19601970 », Histoire@Politique, [en ligne], nº 30, septembre-décembre 2016, www.histoire-politique.fr

réalités existantes (comme la communauté de l'Isolotto, dans la banlieue de Florence, une paroisse fortement engagée dans les luttes ouvrières et sociales, à plusieurs reprises condamnée par l'archevêque ${ }^{5}$ ) ou, dans d'autres, sur de nouveaux types de rassemblement, essentiellement composés de jeunes, qui débouchent sur des manifestations de protestation d'une ampleur encore inconnue en Italie (c'est le cas de la prédication du contro-quaresimale sur le parvis de la cathédrale de Trente ou de l'occupation de la cathédrale de Parme en 1968, qui est évacuée par la police).

Les événements de 1968 exacerbent et font littéralement éclater les tensions accumulées au sein du catholicisme italien ${ }^{6}$. Bien avant d'autres universités, c'est dès le mois de novembre 1967 que l'Université catholique de Milan est l'objet de désordres auxquels la police riposte par la dispersion brutale des manifestants. L'explosion de la contestation catholique, de 1968 à 1972, s'accompagne de la naissance de nouveaux groupes et d'une tension croissante à l'égard des institutions ecclésiastiques7. Beaucoup de ces groupes ont une courte durée de vie ; certains, au contraire, sont encore actifs (comme la communauté de Bose, en Piémont, fondée par Enzo Bianchi, qui est devenue un monastère œcuménique mixte, où vivent des moines et des moniales). Un grand nombre de ces regroupements se caractérisent par la volonté d'affirmer l'unité (et, même, certaines fois, l'identification) entre condition ouvrière et expérience chrétienne, comme dans le cas des prêtres-ouvriers ${ }^{8}$ et du réseau des ACLI, l'organisation de masse des travailleurs catholiques qui se distingue par son spectaculaire "choix socialiste ${ }^{9}$ ». Il faut souligner l'importance de la décision des syndicalistes appartenant à la FIM-CISL (la Fédération des travailleurs de la métallurgie de la Confédération italienne des syndicats des travailleurs, en grande partie composée de catholiques) de soutenir l'unité d'action des organisations " démocratiques » des travailleurs (par le rapprochement avec la CGIL communiste et la UIL socialiste) qui ira même jusqu'à modifier les équilibres internes de l'ensemble de la confédération ${ }^{10}$.

D'autres groupes se concentrent sur la critique du célibat ecclésiastique, de la morale catholique, du cléricalisme et de l'autoritarisme au sein de l'Église, comme la communauté du Vandalino de Turin, où, en 1970, l'abbé Vittorino Merinas célèbre le mariage de deux prêtres avec deux jeunes femmes, dont l'une est une religieuse, ce qui provoque la ferme condamnation du cardinal Michele Pellegrino, qui est pourtant

5 Cf. Christian G. De Vito, Mondo operaio e cristianesimo di base. L'esperienza dell'Isolotto di Firenze, Rome, Ediesse, 2011

${ }^{6}$ Cf. Guido Verucci, « Il '68, il mondo cattolico italiano e la Chiesa », dans Aldo Agosti, Luisa Passerini et Nicola Tranfaglia (dir.), La cultura e i luoghi del '68, Milan, F. Angeli, 1991, p. 381-403; Agostino Giovagnoli (dir.), 1968 : fra utopia e Vangelo, Rome, AVE, 2000.

7 Cf. Giovanni Bianchi, L'Italia del dissenso, Brescia, Queriniana, 1969, p. 59-72.

${ }^{8}$ Cf. Maurilio Guasco, « Preti-operai », dans Francesco Traniello et Giorgio Campanini (dir.), Dizionario storico del movimento cattolico in Italia. 186o-1980, tome I/2, Turin, Marietti, 1981, p. 366-373 ; Pietro Crespi, Prete operaio. Testimonianze di una scelta di vita, Rome, Edizioni Lavoro, 1985; Marta Margotti, Lavoro manuale e spiritualità. L'itinerario dei preti operai, Rome, Studium, 2001.

9 Cf. Fausto Tortora, "Le ACLI e la scelta socialista », dans I cristiani nella sinistra. Dalla resistenza a oggi, Rome, Coines, 1976, p. 199-213 ; Maria Cristina Sermanni, Le ACLI alla prova della politica. 19611972, Naples, Edizioni Dehoniane, 1986 ; Alberto Scarpitti et Carlo Felice Casula (dir.), L’ipotesi socialista. Trent'anni dopo. 1970-200o, Rome, Aesse, 2001.

${ }^{10}$ Cf. Carlo Felice Casula, «Le ACLI e la CISL negli anni Settanta : pratiche sociali e tentazioni della politica », dans Francesco Malgeri et Leonardo Paggi (dir.), L'Italia repubblicana nella crisi degli anni Settanta, tome 3 : Partiti e organizzazioni di massa, Soveria Mannelli, Rubbettino, 2003, p. 169-188; Paolo Trionfini, La laicità della CISL. Autonomia e unità sindacale negli anni Sessanta, Brescia, Morcelliana, 2014. 
Marta Margotti, « À la gauche de Dieu. Une géographie de la contestation catholique en Italie dans les années 19601970 », Histoire@Politique, [en ligne], nº 30, septembre-décembre 2016, www.histoire-politique.fr

l'un des rares évêques italiens disposés au dialogue avec les contestataires. C'est à partir de 1968 que l'on voit la naissance de plusieurs tentatives de coordination entre les revues de la dissidence, les communautés de base et les groupes spontanés ${ }^{11}$, essentiellement pour contrer certains choix privilégiés par Paul VI (en particulier, dans les encycliques Sacerdotalis Cælibatus en 1967 et Humanae Vitae en 1968) ${ }^{12}$ et les prises de position virulentes de certains évêques contre les « contestataires » (par exemple, les mesures adoptées à Florence par le cardinal Ermenegildo Florit, à Gênes par le cardinal Giuseppe Siri contre Agostino Zerbinati, curé de la paroisse d'Oregina, ou à Rome contre dom Giovanni Franzoni, l'abbé du monastère bénédictin de SaintPaul-hors-les-murs, suspendu a divinis pour son soutien à la loi sur le divorce) ${ }^{13}$. Ces groupes diffusent, de 1968 à 1974, un Bollettino di collegamento fra comunità cristiane in Italia (Bulletin de liaison des communautés chrétiennes en Italie).

Les difficultés rencontrées pour fédérer de semblables expériences sont dues au caractère hétérogène des motivations et des objectifs présents dans les différents groupes, mais également à l'effritement progressif de la contestation. Après 1972, plus que les tentatives de recomposition de la mouvance catholique (comme celle qu'avait proposée le jésuite Bartolomeo Sorge ${ }^{14}$, directeur de l'influente revue $\mathrm{La}$ Civiltà Cattolica), c'est l'éloignement de « l'esprit de 68 » qui conditionne l'évolution des expériences se réclamant de cette référence. Si l'on exclut la cohésion retrouvée à l'occasion du référendum de 1974 (notamment lorsqu'ils défendent la loi sur le divorce), comme autour des demandes de réforme du concordat, ou pour la paix au Vietnam, ou encore pour s'opposer aux compromissions des institutions catholiques avec les gouvernements autoritaires d'Europe et d'Amérique Latine, les catholiques de la dissidence les plus politiquement engagés sont souvent responsables d'une fragmentation au sein des multiples formations politiques surgies à la gauche $\mathrm{du}$ Parti communiste ${ }^{15}$. La question de l'engagement antifasciste et de la "violence révolutionnaire » anime les discussions de nombre de communautés de base en Italie qui, souvent, se réfèrent à la pensée et à l'action du prêtre bolivien Camilo Torres et sont attirées par le mythe de Che Guevara. C'est également à la suite de ces réflexions que certains militants catholiques se mobilisent dans des groupuscules d'extrême gauche, même s'il est difficile de définir précisément un lien direct entre légitimation chrétienne de la violence parfois élaborée dans ces milieux et participation à l' " action directe» de certains fidèles contestataires. Néanmoins, rares sont les exemples de passage de catholiques contestataires à la clandestinité et à la lutte armée (comme Margherita Cagol et Renato Curcio, parmi les fondateurs des Brigades Rouges) ${ }^{16}$. Cependant, la diffusion de réflexions sur le rapport entre foi

${ }^{11}$ Cf. Sergio Ristuccia (dir.), Intellettuali cattolici tra riformismo e dissenso. Polemiche sull'integrismo, obbedienza e fine dell'unita politica, rifiuto dell'istituzione nelle riviste degli anni Sessanta, Milan, Edizioni di Comunità, 1975 ; Gino Benvenuti, Informare $i$ cattolici. La rivista "Il Regno" 1966-1971, Reggio Emilia, Bonhoeffer, 1980 ; Carmelo Adagio, « Le riviste del dissenso cattolico », Parolechiave, tome $40, \mathrm{n}^{\circ} 18,1998$, p. 119-138.

${ }^{12}$ Cf. Fulvio De Giorgi, Paolo VI. Il papa del moderno, Brescia, Morcelliana, 2015, pp. 451-602.

13 Cf. Virgilio Levi (dir.), Di fronte alla contestazione. Testi di Paolo VI, Milan, Rusconi, 1970 ; Andrea Riccardi, Il potere del papa da Pio XII a Giovanni Paolo II, Rome-Bari, Laterza, 1993; Comunità dell'Isolotto, Oltre $i$ confini. Trent'anni di ricerca comunitaria, Florence, Libreria Editrice Fiorentina, 1995 ; Giovanni Franzoni, Autobiografia di un cattolico marginale, Soveria Mannelli, Rubbettino, 2014. ${ }^{14}$ Cf. Bartolomeo Sorge, La ricomposizione dell'area cattolica in Italia, Rome, Città nuova, 1979.

15 Cf. Lorenzo Bedeschi, Cattolici e comunisti. Dal socialismo cristiano ai cristiani marxisti, Milan, Feltrinelli, 1974.

${ }^{16}$ Cf. Guido Panvini, Cattolici e violenza politica. L'altro album di famiglia del terrorismo italiano, Venice, Marsilio, 2014. 
Marta Margotti, «À la gauche de Dieu. Une géographie de la contestation catholique en Italie dans les années 19601970 », Histoire@Politique, [en ligne], n 30, septembre-décembre 2016, www.histoire-politique.fr

chrétienne et violence favorise la participation de beaucoup de prêtres et de laïcs aux mouvements pacifistes et antimilitaristes, certains avec une évidente origine religieuse, comme dans le cas de Pax Christi, le mouvement international né en 1954 qui en Italie organise, dans les années de la contestation, de nombreuses manifestations contre la guerre en Vietnam et pour l'objection de conscience au service militaire.

Quoi qu'il en soit, toutes les tentatives émanant de certaines de ces formations catholiques de gauche pour se présenter aux élections politiques nationales se révèlent des échecs, comme c'est le cas en 1972 pour le MPL, le Mouvement politique des travailleurs : fondé deux ans avant par l'ancien président des ACLI Livio Labor, le MPL se veut un sujet politique progressiste, contraire au conservatisme de la Démocratie chrétienne et à l'organisation capitaliste du travail, mais, après la défaite électorale, le Mouvement décide de se dissoudre. Et c'est précisément parce qu'ils ne sont en aucun cas réductibles à l'histoire interne de chacune des organisations politiques en présence que les groupes catholiques les plus attentifs à la dimension religieuse et spirituelle (où les revendications de réforme de l'Église demeurent importantes) parviennent en revanche à donner une continuité majeure à leur action et à maintenir une identité communautaire plus clairement définie, souvent liée à une situation locale particulière ou à des expériences d'engagements sociaux (comme celles pour la réinsertion des toxicomanes menées par le Gruppo Abele de Turin, fondé par l'abbé Luigi Ciotti, qui obtint constamment le soutien du cardinal Michele Pellegrino, ou par la communauté de San Benedetto al Porto de Gênes, animée par l'abbé Andrea Gallo) ${ }^{17}$.

À partir de la fin des années 1970, plus qu'à un déclin de la contestation catholique, on assiste à sa transformation. Après le regain éphémère du mouvement de la jeunesse de l'année 1977, le changement intervenu à l'intérieur de la dissidence catholique est symbolisé, en premier lieu, par l'enlèvement et l'assassinat par les Brigades rouges, au printemps 1978, du secrétaire de la Démocratie chrétienne, le catholique Aldo Moro, qui tentait alors de réaliser une opération complexe d'implication du Parti communiste italien au sein du gouvernement - connue sous la fameuse appellation de « compromis historique ». Survient en second lieu, peu après, la disparition de Paul VI. C'est ainsi que s'achève une phase, à la fois brève et intense, riche en initiatives, en projets et en espérances qui sera bientôt suivie d'une autre, tout aussi incertaine, mais d'une nature plus tourmentée encore. Les « catholiques désobéissants » se trouvent en quelque sorte piégés entre le dynamisme de nouveaux mouvements, considérés comme des éléments stratégiques au sein de l'Église de Jean-Paul II (comme Comunione e liberazione - alors qu'au début des années 1970 il avait certaines références à la pensée progressiste, il devient ensuite l'un des mouvements catholiques les plus politiquement conservateurs et les plus fidèles à la hiérarchie papale), et le reflux culturel et politique enregistré dans la société italienne où les initiatives sollicitant la participation active des citoyens et la mobilisation par le bas trouvent toujours moins de possibilités de s'exprimer. Moins visible sur la scène médiatique et plus attentive à la dimension biblique et communautaire, la constellation de la dissidence catholique enregistre une certaine stabilisation de ses assises internes, avec une concentration croissante sur les questions du pacifisme, de la solidarité internationale et de l'intégration sociale.

${ }_{17}$ Cf. Giuseppe Alberigo (dir.), Chiese italiane e Concilio. Esperienze pastorali nella Chiesa italiana tra Pio XII e Paolo VI, Gênes, Marietti, 1988. 
Marta Margotti, «À la gauche de Dieu. Une géographie de la contestation catholique en Italie dans les années 19601970 », Histoire@Politique, [en ligne], nº 30, septembre-décembre 2016, www.histoire-politique.fr

\section{Une carte géographique et culturelle de la gauche catholique}

La rapidité des transformations sociales et culturelles des années 1960 et 1970 se reflète dans l'articulation interne de la gauche catholique qui, à son tour, du moins en partie, conditionne les principaux changements alors en cours dans la société italienne. Malgré les différences notables d'origine et d'objectifs, les diverses expériences de la contestation catholique italienne ont en commun la lecture directe et communautaire de la Bible ainsi que la tension croissante avec les institutions ecclésiastiques et l'engagement auprès des plus démunis ${ }^{18}$. La carte de l'Italie de la dissidence révèle une tendance à la concentration des expériences dans les grands centres urbains et au sein des régions situées au centre-nord de la péninsule. Dans ces territoires, le développement de formes de contestation à l'intérieur des structures catholiques de masse, ainsi que la naissance de nombreuses communautés de base, sont favorisés par un contrôle social moins fort, une plus large circulation d'idées religieuses "non conformistes" ainsi que la présence d'expériences échappant à l'organisation ecclésiastique traditionnelle des paroisses ; dans les villes, en outre, les militants catholiques perçoivent de plus en plus clairement la diffusion des phénomènes de sécularisation et peuvent établir des contacts avec des mouvements ouvriers et des cercles intellectuels ${ }^{19}$, comme par exemple à Milan, Rome, Florence, Gênes, Turin et Brescia. Ces groupes rassemblent essentiellement des étudiants provenant des classes moyennes, des employés, des enseignants, mais aussi, pour une proportion non négligeable, des ouvriers. La dimension intellectuelle est fortement présente, aussi bien à travers le choix d'un approfondissement théologique et biblique que par le biais de l'étude des textes de la culture socialiste et, plus particulièrement, de la pensée marxiste (souvent par la médiation des livres du philosophe Roger Garaudy et, surtout, du prêtre salésien Giulio Girardi ${ }^{20}$ ).

En moins grand nombre, des groupes appartenant à la mouvance de la dissidence catholique naissent également au sein de petits centres urbains de l'Italie du Sud et des zones rurales où les traditions, notamment religieuses, sont plus puissamment enracinées. C'est précisément en raison de leur localisation géographique et sociale que ces communautés parviennent à impliquer à la fois des paysans, des femmes au foyer et des personnes âgées, par le biais de modes de participation moins fortement intellectualisés, mais avec un intérêt croissant pour les problèmes sociaux et une valorisation de certains aspects de la religiosité populaire. L'étroite connexion entre la contestation politique et religieuse au sein des communautés du Sud de l'Italie provoque d'ailleurs certaines sanctions virulentes à leur encontre de la part des évêques, mais aussi l'écoute des partis politiques de gauche, attirés par ces formes inédites de mobilisation populaire. C'est le cas, entre autres, de la paroisse du Carme à Conversano, dans les Pouilles, où en 1970 le curé est éloigné par l'évêque pour ses choix pastoraux jugés trop progressistes, de la communauté de l’abbé Marco Bisceglia

${ }^{18} \mathrm{Cf}$. Fausto Perrenchio, Bibbia e comunità di base in Italia, Rome, LAS, 1980.

19 Cf. Arnaldo Nesti (dir.), L'altra Chiesa in Italia, Milan, A. Mondadori, 1970 ; Gian Enrico Rusconi et Chiara Saraceno, Ideologia religiosa e conflitto sociale, Bari, De Donato, 1970 ; Franco Ferraresi, Anna Lena, Giorgio Ferraresi, Bruno Manghi et Franco Rositi, La politica dei gruppi, Milan, Edizioni di Comunità, 1970 ; Arturo Parisi, " La matrice socio-religiosa del dissenso cattolico in Italia », Il Mulino, tome $20, \mathrm{n}^{\circ} 4,1971$, p. 637-657.

${ }^{20}$ Cf. parmi les nombreux livres publiés par Girardi, engagé dans le mouvement des Chrétiens pour le socialisme, Marxismo e cristianesimo, Assise, Cittadella, 1973. 
Marta Margotti, «À la gauche de Dieu. Une géographie de la contestation catholique en Italie dans les années 19601970 », Histoire@Politique, [en ligne], n 30, septembre-décembre 2016, www.histoire-politique.fr

à Lavello, en Basilicate, qui en 1974 est suspendu a divinis, et de la communauté de Gioiosa Ionica en Calabre, active surtout depuis 1973, qui a dénoncé l'enchevêtrement des affaires des institutions religieuses et de la Démocratie chrétienne et les liens entre certains prêtres et la mafia.

\section{Pour une histoire connectée de la gauche catholique}

Les influences et les contacts internationaux qui se développent fréquemment dans les groupes de la dissidence italienne des années 1960 et 1970 sont un élément nouveau primordial, qui influe de manière décisive sur les réflexions et les stratégies de mobilisation. La prise de conscience du fait d'appartenir à un mouvement de contestation (catholique, mais pas uniquement) de dimension planétaire, l'imitation de formes de protestation pratiquées à l'étranger, l'attraction exercée par certains courants théologiques et politiques expérimentés dans le nord de l'Europe et en Amérique latine, la rencontre avec des leaders chrétiens de gauche de passage en Italie, mais également les voyages à l'étranger et les échanges épistolaires avec des missionnaires permettent à de nombreux prêtres et laïcs de rompre avec le tropisme romain traditionnel qui prédominait dans l'Église italienne. Les références à Mounier et Maritain sont plutôt rares (contrairement à ce qui passait dans les cercles du progressisme catholique des années 1930-1950) et il en est de même pour l'invocation du renouveau promu par les mouvements œcuménique et liturgique de la première moitié du $\mathrm{XX}^{\mathrm{e}}$ siècle. On observe, en revanche, l'influence des expériences et des réflexions latino-américaines (surtout par l'entremise des publications liées à la théologie de la libération, au mouvement des Chrétiens pour le socialisme et aux communautés de base ${ }^{21}$ ), de même que celle des travaux des théologiens nordaméricains et allemands (en particulier la théologie de la sécularisation et les écrits de Dietrich Bonhoeffer, la théologie radicale de Harvey Cox, la théologie politique de Johann Baptist Metz, les livres de Hans Küng ${ }^{22}$ ). On note aussi l'écho des débats

\footnotetext{
${ }^{21}$ Voir par exemple José Maria González Ruiz, Il cristianesimo non è un umanesimo. Appunti per una teologia del mondo, préf. de Marie-Dominique Chenu, Assise, Cittadella, 1967, trad. de El cristianismo no es un humanismo. Apuntes para una teología del mundo, Barcelona, Península, 1966 ; Hélder Câmara, Rivoluzione nella pace, Milan, Jaca Book, 1968, trad. de Revolução dentro da paz, Rio de Janeiro, Sabiá, 1968, éd. fr. Révolution dans la paix, Paris, Seuil, 1970 ; Arturo Paoli, Dialogo della liberazione, Brescia, Morcelliana, 1969 (préf. Sergio Soave, Turin, Aragno, 2012) ; Gustavo Gutiérrez, Teologia della liberazione. Prospettive, Brescia, Queriniana, 1972, trad. de Teología de la liberación. Perspectivas, Lima, CEP, 1971, éd. fr. Théologie de la libération. Perspectives, Paris-Bruxelles, Lumen Vitae, 1974; Leonardo Boff, Gesù Cristo liberatore, Assise, Cittadella, 1973, trad. de Jesus Cristo libertador, Petropólis, Vozes, 1972 ; éd. fr. Jésus-Christ libérateur, Paris, Cerf, 1974.

${ }^{22}$ Cf. Harvey Cox, La città secolare, Florence, Vallecchi, 1968, trad. de The Secular City. Secularization and Urbanization in Theological Perspective, New York, Macmillan, 1965, éd. fr. La cité séculière. Essai théologique sur la sécularisation et l'urbanisation, Tournai, Casterman, 1965; Johann Baptist Metz, Sulla teologia del mondo, Brescia, Queriniana, 1969, trad. de Zur Theologie der Welt, Mainz-München, Matthias-Grünewald-Kaiser, 1968, éd. fr. Pour une théologie du monde, Paris, Cerf, 1971; Dietrich Bonhoeffer, Resistenza e resa. Lettere e appunti dal carcere, Milan, Bompiani, 1969, trad. de Widerstand und Ergebung. Briefe und Aufzeichnungen aus der Haft, München, Kaiser, 1964, éd. fr. Résistance et soumission. Lettres et notes de captivité, Genève, Labor et Fides, 1967; Hans Küng, La Chiesa, Brescia, Queriniana, 1969, trad. de Die Kirche, Freiburg im Breisgau, Herder, 1967, éd. fr. L’Église, Paris, Desclée de Brouwer, 1968.
} 
Marta Margotti, «À la gauche de Dieu. Une géographie de la contestation catholique en Italie dans les années 19601970 », Histoire@Politique, [en ligne], n 30 , septembre-décembre 2016, www.histoire-politique.fr

autour du catéchisme hollandais ${ }^{23}$, de l'expérience des prêtres-ouvriers et du dialogue entre chrétiens et communistes en France ${ }^{24}$, mais aussi des textes des pères Chenu et Congar ${ }^{25}$. De nombreux ouvrages et articles relatifs à la contestation catholique sont traduits, entraînant la création de plusieurs collections éditoriales consacrées à ces sujets ${ }^{26}$.

Parallèlement, les écrits et les expériences de la contestation catholique italienne connaissent une certaine notoriété à l'étranger, comme le montrent les traductions de nombreux livres ainsi que les contacts et les voyages de prêtres et militants hors d'Italie ${ }^{27}$. Ce même courant de pensée devient, également en Italie, une caisse de résonance pour les luttes contre la guerre du Vietnam et contre les dictatures « catholiques » en Espagne ou au Portugal, et pour la dénonciation de la proximité de l'Eglise avec les régimes autoritaires d'Amérique latine. C'est ainsi que se crée un nouveau regard sur les missions, considérées désormais comme des expressions de l'impérialisme occidental, mais aussi comme une voie de sortie du conservatisme religieux européen : les problématiques du sous-développement s'insèrent alors dans le cadre plus général du combat contre le système capitaliste et en faveur de la libération des peuples. De nombreux débats sur l'œcuménisme sont organisés en Italie, ainsi que certains contacts avec les chrétiens des pays d'Europe de l'Est, mais plus rarement avec l'« Église du silence », soupçonnée de nourrir un préjugé anticommuniste insurmontable.

Cette poussée vers la globalisation religieuse, alimentée par la dissidence et également favorisée en Italie par le passage de nombreux représentants de la gauche catholique se rendant le plus souvent à Rome, est un signe caractéristique de ce cycle de bouleversements et, peut-être, l'un de ses héritages les plus durables ${ }^{28}$.

\footnotetext{
${ }^{23}$ Il dossier del Catechismo olandese, textes recueillis par Aldo Chiaruttini, notes historique et théologiques de Léo Alting von Geusau et Fernando Vittorino Joannes, Milan, A. Mondadori, 1968, éd. fr. Le dossier du catéchisme hollandais. Un nouveau langage théologique, Paris, Fayard, 1969.

24 Cf. la traduction de certains ouvrages de Roger Garaudy: par exemple, Prospettive dell'uomo. Esistenzialismo, cattolicesimo, strutturalismo, marxismo, Turin, Borla, 1972, trad. de Perspectives de l'homme. Existentialisme, pensée catholique, structuralisme, marxisme, Paris, Presses universitaires de France, 1969 ; Dall'anatema al dialogo (contient aussi : Johannes Baptist Metz, Risposta a Garaudy), Brescia, Queriniana, 1969, trad. de De l'anathème au dialogue. Un marxiste s'adresse au Concile, Paris, Plon, 1965).

25 Parmi les nombreux ouvrages des deux théologiens dominicains traduits en italien, voire MarieDominique Chenu, Il Vangelo nel tempo, Rome, AVE, 1968, trad. de La parole de Dieu, tome 2 : L'Évangile dans le temps, Paris, Cerf, 1964; Yves Congar, Una Chiesa contestata. Meditazioni teologiche, Brescia, Queriniana, 1969, trad. de Au milieu des orages. L'Église affronte aujourd'hui son avenir, Paris, Cerf, 1969.

${ }^{26}$ Cf. Lucia Ceci, « L'editoria cattolica nel periodo postconciliare. Il caso della Queriniana », Annali della Fondazione Luigi Einaudi, tome 30, n 1, 1996, p. 393-431.

27 Le livre de Girardi, Marxismo e cristianesimo, cité, a été traduit en français, anglais, allemand, portugais, espagnol, catalan et japonais. En France, sont également parus Comunità dell'Isolotto, Le dossier de l'Isolotto, introduction d'Enzo Mazzi, Paris, Seuil, 1970, trad. de Isolotto, 1954-1969, Bari, Laterza, 1969; Giovanni Franzoni, La terre appartient à Dieu. Lettre pastorale, avec une postface de Robert Gantoy, Paris, Centurion, 1973, trad. de La terra è di Dio. Lettera pastorale, Rome, COM, 1973 ; Ernesto Balducci, La foi ne sert à rien. Une nouvelle identité chrétienne, Paris, Cerf, 1976, trad. de La fede dalla fede. Meditazioni sulla nuova identità cristiana, Assise, Cittadella, 1975.

${ }^{28}$ Cf. Gerd-Rainer Horn, The Spirit of Vatican II. Western European Progressive Catholicism in the Long Sixties, Oxford, Oxford University Press, 2015.
} 
Marta Margotti, « À la gauche de Dieu. Une géographie de la contestation catholique en Italie dans les années 19601970 », Histoire@Politique, [en ligne], nº 30, septembre-décembre 2016, www.histoire-politique.fr

L'observation de cet archipel de la contestation catholique italienne permet de formuler, en conclusion, quelques remarques sur les rapports entre les phénomènes de sécularisation, les dynamiques sociales et les transformations de l'Église postconciliaire. Dans la péninsule, les ferments de réforme religieuse qui, jusqu'au début des années 1960, n'avaient concerné que des groupes circonscrits de fidèles, se sont développés rapidement avec le concile et les mouvements de protestation sociale. L'imbrication complexe des éléments présents dans la contestation catholique a favorisé la radicalisation des positions, plus accentuée dans les territoires où les traditions culturelles et le contrôle social exercé par l'Église étaient plus importants et où les autorités ecclésiastiques manifestaient une fermeté sans appel vis-à-vis de la dissidence. Même si on constate des différences notables également dans l'attitude des évêques italiens, comme dans la curie romaine ${ }^{29}$, il s'agissait, pour la plus grande partie de l'épiscopat, d'endiguer la présence d'idées politiques et religieuses considérées comme subversives, mais également de défendre la vision d'une " nation catholique » où l'Église devait continuer à jouer un rôle central ${ }^{30}$. Au contraire, pour les catholiques de gauche, la crise du christianisme dans une Italie désormais largement sécularisée imposait à l'Église de changer radicalement de méthodes et d'objectifs (notamment en ce qui concernait la théologie, la liturgie ainsi que les relations entre Église et pouvoir politique), en acceptant et en dépassant l'aggiornamento du concile. La superposition entre identité ouvrière et identité chrétienne, affirmée dans de nombreux groupes de la dissidence, tentait, au-delà de la réélaboration de suggestions récurrentes dans les mouvements de protestation des années 1960 et 1970, de proposer une alternative à la crise du christianisme dans la société industrielle. Cependant, la plupart de ces groupes dissidents ont échoué à réaliser leur projet: la capacité de médiation limitée, l'intellectualisme de leurs propositions et l'élitisme d'un grand nombre d'initiatives rendaient impossibles l'implication des «masses catholiques» et, plus encore, celle des «masses populaires ». La lutte contre les structures de pouvoir de l'Église, le combat sans concession pour une révolution religieuse et politique, ainsi que la très forte conscience réflexive de beaucoup de catholiques contestataires (qui sont nombreux à témoigner publiquement de leur expérience et de leur itinéraire ${ }^{31}$ ), jalonnent la contestation dans une Église italienne qui, même dans ses composantes les plus réfractaires au changement, est sortie irrémédiablement métamorphosée de cette période historique.

(traduit de l'italien par Anne-Catherine Caron)

\footnotetext{
${ }^{29}$ Alessandro Santagata, La contestazione cattolica..., op. cit., p. 223-230.

$3^{\circ} \mathrm{Cf}$. Andrea Riccardi, «La nazione cattolica », dans Agostino Giovagnoli (dir.), Interpretazioni della Repubblica, Bologne, Il Mulino, 1998, p. 47-61; Marco Impagliazzo (dir.), La nazione cattolica. Chiesa e società in Italia dal 1958 a oggi, Milan, Guerini e Associati, 2004 ; Francesco Traniello, Religione cattolica e Stato nazionale. Dal Risorgimento al secondo dopoguerra, Il Mulino, Bologna 2007.

${ }^{31}$ Cf. Vittorino Merinas (dir.), Dossier sulla contestazione nella Chiesa, Turin, Gribaudi, 1969 ; Marco Boato (dir.), Contro la Chiesa di classe. Documenti della contestazione ecclesiale in Italia, Padova, Marsilio, 1969; Nando Fabro, I cattolici e la contestazione in Italia, Fossano, Esperienze, 1970; Comunità dell'Isolotto, Isolotto sotto processo, Bari, Laterza, 1971; Bruno D'Avanzo, Tra dissenso e rivoluzione. Gruppi cristiani a Firenze, préf. de Lidia Menapace, Bologne, Guaraldi, 1971 ; Peppino Orlando, La comunità di Oregina. Evangelo e marxismo nel dissenso cattolico, Turin, Claudiana, 1973 ; Roberto Sciubba et Rossana Sciubba Pace, Le comunità di base in Italia, 2 tomes, Rome, Coines, 1976 ; Massa e Meriba. Itinerari di fede nella storia delle comunità di base, Turin, Claudiana-Tempi di fraternità, 1980 ; Mario Cuminetti, Il dissenso cattolico in Italia. 1965-198o, Milan, Rizzoli, 1983; Uomini di frontiera. Scelta di classe e trasformazioni della coscienza cristiana a Torino dal Concilio a oggi, Turin, Coop. di cultura Lorenzo Milani, 1984.
} 
Marta Margotti, «À la gauche de Dieu. Une géographie de la contestation catholique en Italie dans les années 19601970 », Histoire@Politique, [en ligne], n 30 , septembre-décembre 2016, www.histoire-politique.fr

\title{
L'auteur
}

Marta Margotti est professeur d'histoire contemporaine à l'Université de Turin. Ses travaux portent sur l'histoire sociale, l'histoire du journalisme et l'histoire du christianisme en Italie et en France.

\section{Résumé}

Cette étude élabore une cartographie de la contestation catholique politique et religieuse en Italie dans les années 1960 et 1970. Elle propose une périodisation de la dissidence catholique, un panorama à la fois topographique et culturel des mouvements chrétiens de gauche et un aperçu des rapports internationaux tissés par les différents groupes en présence. Certaines considérations permettent une comparaison entre la contestation catholique propre à l'Italie et les expériences accomplies dans d'autres pays, ce qui conduit à esquisser, en conclusion, une contribution à l'élaboration d'une histoire globale du progressisme catholique.

Mots clés : catholicisme ; progressisme catholique ; contestation ; gauche ; Italie.

\begin{abstract}
This article presents a survey of Catholic political and religious unrest in Italy in the 1960 s and 70s. It offers a periodization of Catholic dissidence, a simultaneously topographical and cultural overview of leftwing Christian movements and an assessment of the international links established by the various groups concerned. Some of these considerations allow specifically Italian Catholic activism to be compared with the experiences of other countries, thereby contributing to the development of a global history of Catholic progressivism.
\end{abstract}

Key words: Catholicism; Catholic Progressivism; Protest; Left; Italy.

Pour citer cet article : Marta Margotti, «À la gauche de Dieu. Une géographie de la contestation catholique en Italie dans les années 1960-1970 ", Histoire@Politique, [en ligne], $\mathrm{n}^{\circ} 30$, septembre-décembre 2016, www.histoire-politique.fr 


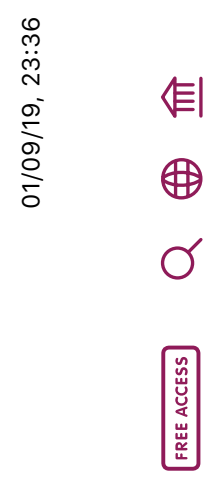

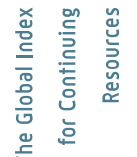

क止
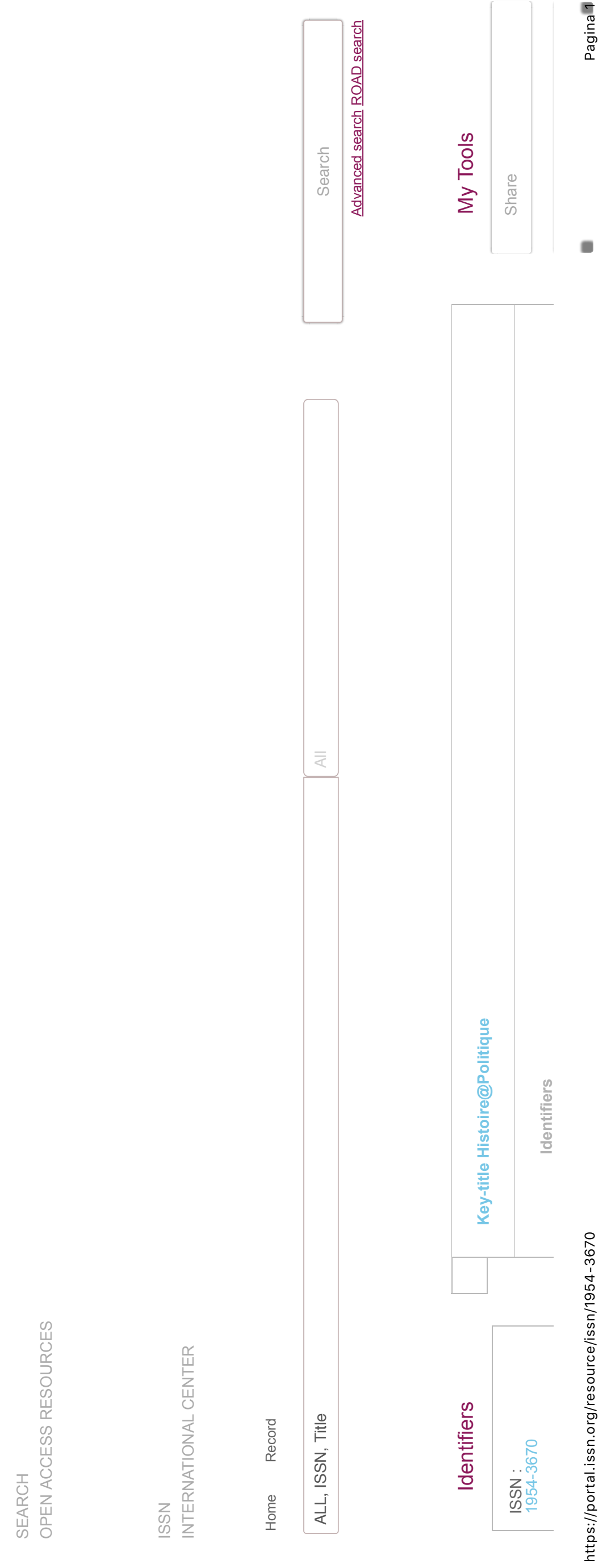


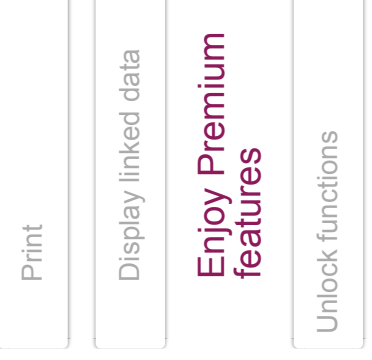

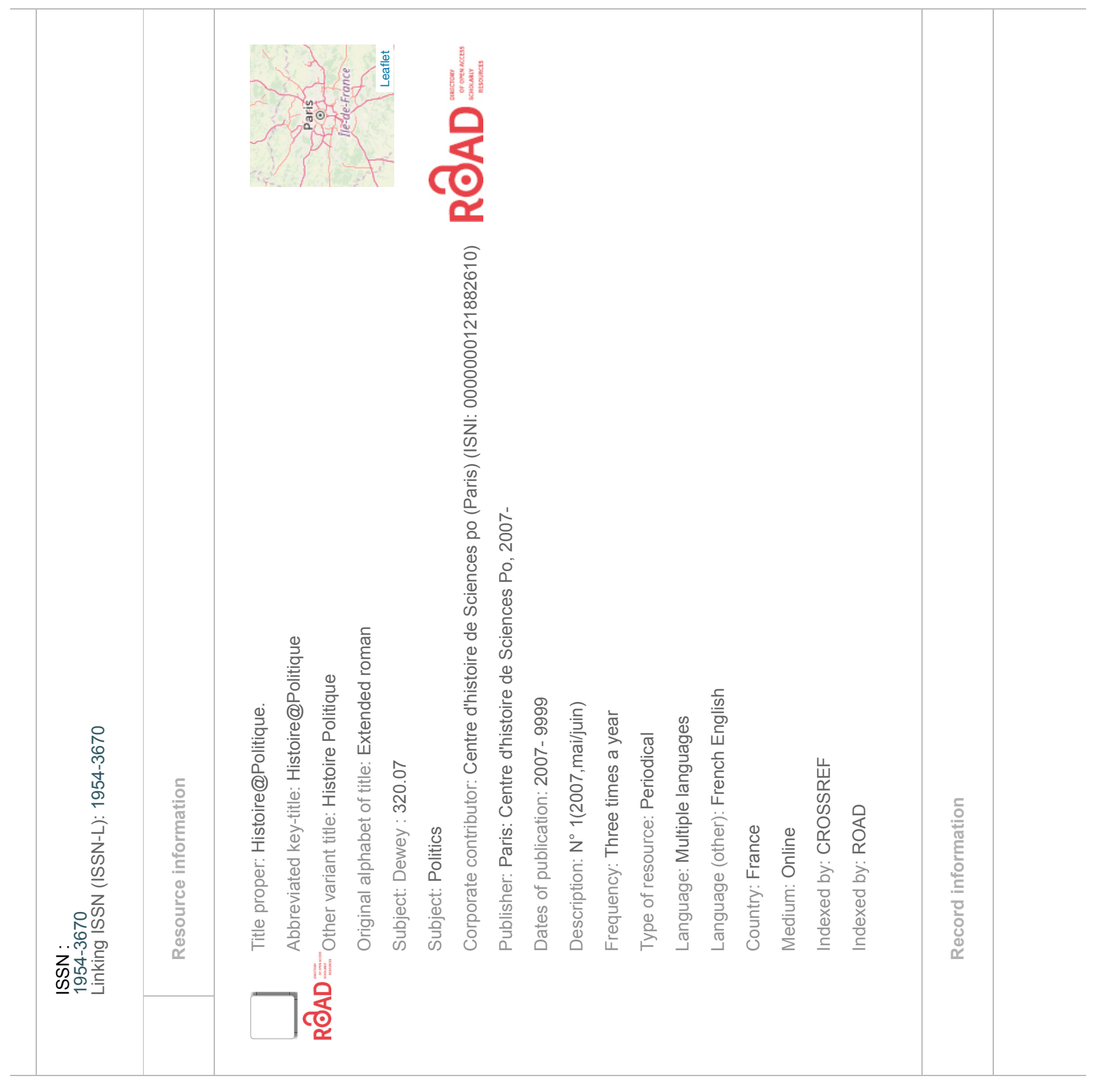




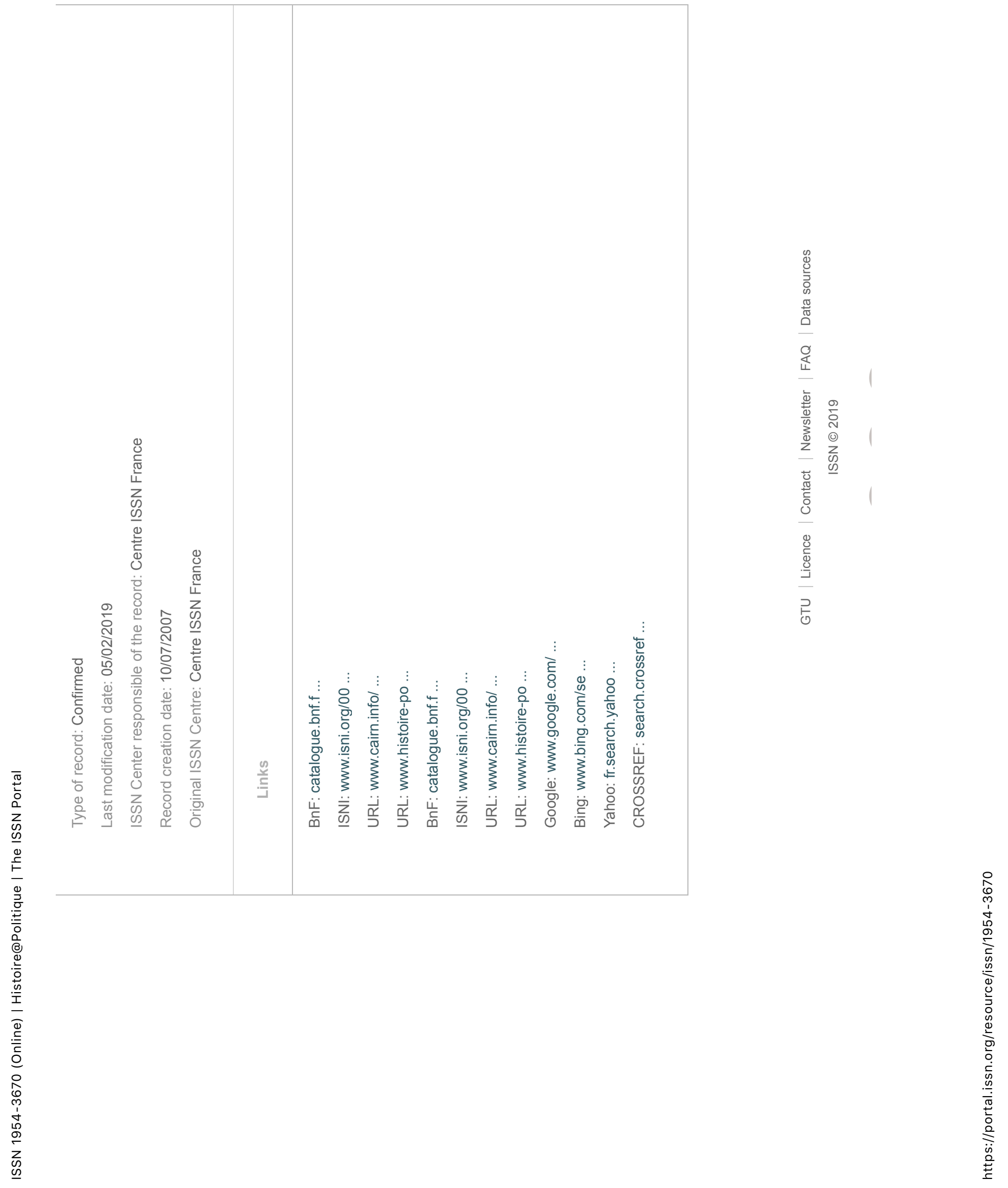

